Notes

\title{
High-Performance Liquid Chromatography with Chemiluminescence Detection of Methamphetamine and Amphetamine in Human Urine
}

\author{
Kenichiro NaKaShima*, Keiko Suetsugu*, Kazunori YoshidA**, Kazuhiro ImaI*** \\ and Shuzo AKIYAma* \\ *School of Pharmaceutical Sciences, Nagasaki University, Nagasaki 852, Japan \\ **Forensic Science Laboratory, Nagasaki Prefectural Police Headquarters, Nagasaki 852, Japan \\ ***Branch Hospital Pharmacy, University of Tokyo, Mejirodai, Bunkyo, Tokyo 112, Japan
}

\begin{abstract}
Keywords Amphetamine, methamphetamine, high performance liquid chromatography, chemiluminescence, $N$-(4aminobutyl)- $N$-ethylisoluminol, urine
\end{abstract}

The abuse of methamphetamine (MP), especially in Japan, is causing serious social problems. For the identification and determination of MP and its metabolite, amphetamine (AP), in illegal circumstances, simple and sensitive methods are needed. In our previous paper, a sensitive high performance liquid chromatographic (HPLC) determination method of MP has been developed by using $N$-(4-aminobutyl)- $N$-ethylisoluminol (ABEI) as a chemiluminogenic labeling reagent; this was applied to the determination of MP in human serum. ${ }^{1}$ In this paper, the authors have applied the method to the determination of MP and its major metabolite, amphetamine (AP), in human urine.

\section{Experimental}

\section{Reagents and materials}

Methamphetamine hydrochloride was obtained from Dainippon Pharmacy (Osaka, Japan) and dissolved in water to give a $10^{-3} \mathrm{M}$ solution. Amphetamine sulfate was synthesized according to the literature ${ }^{2}$ and dissolved in water to give a $10^{-3} \mathrm{M}$. These solutions were diluted to appropriate concentrations with water prior to use.

A $5 \mu \mathrm{mol}$ amount of $N$-(4-aminobutyl)- $N$-ethylisoluminol (ABEI, Tokyo Kasei Kogyo, Tokyo, Japan) in $2 \mathrm{ml}$ of methanol and $5 \mu \mathrm{mol}$ of $N, N^{\prime}$-disuccinimidyl carbonate (DSC, Wako Pure Chemicals, Osaka, Japan) in $2 \mathrm{ml}$ of acetonitrile were mixed in a 10-ml screwcapped test tube and allowed to stand for $2 \mathrm{~h}$ to give an ABEI-DSC reagent solution.

All other reagents used were of analytical reagent grade.

Urine samples from MP addicts were kindly provided for this investigation by the Forensic Science Laboratory of Nagasaki Prefectural Police Headquarters.

\section{Apparatus}

The HPLC system used is the same as that of the previous work. ${ }^{1}$ The system consisted of three HPLC pumps (LC-6A, Shimadzu, Kyoto, Japan), a 7125 injector with a $20 \mu l$ loop (Rheodyne, Cotati, CA, USA), a guard column $(30 \mathrm{~mm} \times 4.6 \mathrm{~mm}$, i.d.) packed with TSK Guardgel ODS-80TM (Tosoh, Tokyo, Japan), a Shimpack CLC-C18 separation column (150×6 mm i.d., particle size $5 \mu \mathrm{m}$, Shimadzu), an R-01 recorder (Rikadenki, Tokyo, Japan) and a lumiphotometer (AL2220 , Atto, Tokyo, Japan) with a spiral flow cell of $60 \mu \mathrm{l}$. Stainless-steel tubing $(0.5 \mathrm{~mm}$ i.d. $)$ was used in all flow lines.

\section{Operating conditions}

The separation of ABEI derivatives of MP and AP was achieved with the mobile phase of methanol-water $(54: 46, \mathrm{v} / \mathrm{v})$ containing $30 \mathrm{mM}$ sodium 1-octanesulfonate (flow rate, $1 \mathrm{ml} / \mathrm{min}$ ). The eluates from separation column were first combined with the flow line of $1.5 \times$ $10^{-2} \mathrm{M}$ potassium ferricyanide in $2.5 \mathrm{M}$ sodium hydroxide (flow rate, $1 \mathrm{ml} / \mathrm{min}$ ) and then mixed with the flow line of $0.3 \mathrm{M}$ hydrogen peroxide containing $10 \mathrm{mM} \beta$ cyclodextrin (flow rate, $1 \mathrm{ml} / \mathrm{min}$ ). The lengths of tubing after the mixing tees were 200 and $100 \mathrm{~mm}$, respectively.

\section{Procedure for determination of $M P$ and $A P$ in urine}

To $2 \mathrm{ml}$ of urine in a 10-ml screw-capped test tube was added $2 \mathrm{ml}$ of $2 \% \mathrm{NaOH}$ under agitation. The solution was added with $2 \mathrm{ml}$ of diethyl ether, vortex mixed for $2 \mathrm{~min}$ and centrifuged at $1000 \times \mathrm{g}$ for $10 \mathrm{~min}$ at $0^{\circ} \mathrm{C}$. The extraction procedure was repeated once more, and the extracts were combined. A $100 \mu \mathrm{l}$ aliquot of the organic layer was transferred to a 5-ml vial and to it were successively added $0.4 \mathrm{ml}$ of methanol, $20 \mu \mathrm{l}$ of $0.5 \%$ triethylamine, and $0.5 \mathrm{ml}$ of ABEI-DSC solution. The mixture was well mixed, heated at $80^{\circ} \mathrm{C}$ for $30 \mathrm{~min}$, chilled with ice-water, and passed through a membrane filter $(0.45 \mu \mathrm{m})$. A $20 \mu \mathrm{l}$ aliquot of the filtrate was subjected to HPLC. 


\section{Results and Discussion}

ABEI is known to be an excellent chemiluminogenic labeling reagent for amines or carboxylic acids. ${ }^{3,4}$ In the previous paper, we applied the reagent to the chemiluminescence detection in HPLC of serum MP. ${ }^{1}$ For pharmaceutical or forensic studies, it is preferable not only to determine MP but also its metabolites. Thus, in this paper, we tried to expand the application of the method to the assay of MP and its metabolite, AP, in urine from the MP addicts. The labeling procedure of MP and AP with ABEI and the HPLC separation procedure for ABEI derivatives were achieved according to the previous method. ${ }^{1}$ In the assay of serum MP, we needed only a deproteinization procedure with acetone for a pretreatment of serum. However, for the assay of MP or AP in urine, an extraction procedure was required, because the labeling reaction should be performed in a nonaqueous medium to increase the reaction yield. Therefore solvent extraction techniques using diethyl ether, chloroform and ethyl acetate were studied. Consequently, diethyl ether was found to be suitable. ${ }^{5}$

Calibration curves of MP and AP with spiked urine were linear up to 5 pmol for MP $(r=0.999)$ and $10 \mathrm{pmol}$ for AP $(r=0.999)$. The lower limits of detection for MP and $\operatorname{AP}(S / N>3)$ were $20 \mathrm{fmol}\left(2 \times 10^{-8} \mathrm{M}\right.$ in urine) and $100 \mathrm{fmol}\left(10^{-7} \mathrm{M}\right.$ in urine) per injection, respectively. By adding each $50 \mu$ lof an aqueous solution of known concentrations of MP $\left(2 \times 10^{-5}, 1 \times 10^{-4}, 2 \times 10^{-4} \mathrm{M}\right)$ and AP $\left(4 \times 10^{-5}, 2 \times 10^{-4}, 4 \times 10^{-4} \mathrm{M}\right)$ to $2 \mathrm{ml}$ of normal human urine, calibration curves for spiked urine were prepared. The percentage recovery of MP or AP was calculated from the slopes of calibration curves obtained from standard solutions and spiked urine. The recoveries for MP and AP were 91 and $67 \%$, respectively. The relative standard deviations of five replicate measurements for $1 \times 10^{-4} \mathrm{M} \mathrm{MP}$ and $2 \times 10^{-4} \mathrm{M}$ AP were 3.9 and $3.3 \%$, respectively. Five urine samples of MP addicts were assayed by the method. As shown in Table 1, AP could be detected together with unchanged MP in three urine samples. The representative HPLC chromatogram was shown in Fig. 1.

The proposed method is very sensitive and can simultaneously determine MP and its metabolite, AP. The sensitivity of the method is higher than those of HPLC-absorptiometry ${ }^{6,7}$ and $\mathrm{GC}^{8,9,10}$ and comparable to that of GC-mass spectrometry ${ }^{11}$, but lower than that of HPLC with peroxyoxalate chemiluminescence detection. ${ }^{5}$ Besides, the method is simpler and more rapid than GC method. 9,10

In conclusion, by only a small modification, the previously developed HPLC method with chemiluminescent detection of MP using ABEI as a chemiluminogenic label was found to be applicable to the determination of MP and AP in urine. The method should be useful for the routine detection of MP or AP in urine of MP addicts.
Table 1 MP and AP contents in urine samples of MP addicts

\begin{tabular}{cccc}
\hline $\begin{array}{c}\text { Urine } \\
\text { No. }\end{array}$ & $\begin{array}{c}\text { Sample } \\
\text { Age(sex) }\end{array}$ & MP( $\left(\mu \mathrm{g} \mathrm{ml}^{-1}\right)$ & AP $\left(\mu \mathrm{g} \mathrm{ml}^{-1}\right)$ \\
\hline 1 & $27(\mathrm{~m})$ & 0.36 & 0.49 \\
2 & $33(\mathrm{~m})$ & 1.10 & 0.19 \\
3 & $41(\mathrm{~m})$ & 0.20 & 0.05 \\
4 & $33(\mathrm{~m})$ & 0.18 & N.D. \\
5 & $47(\mathrm{~m})$ & 0.01 & N.D. \\
\hline
\end{tabular}

N. D.: not detected. (a)

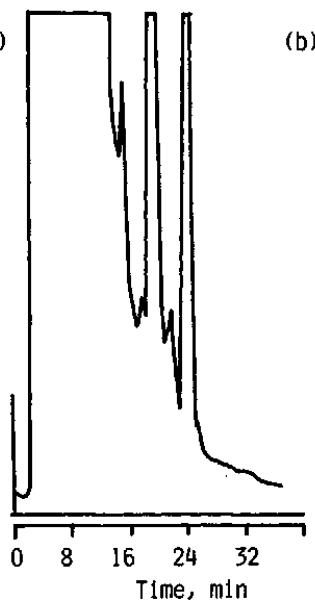

b)



Fig. 1 Chromatograms of ABEI derivatives with MP and AP in urine sample. (a) normal urine, (b) urine of MP addict (sample No. 1 in Table 1); for the other experimental conditions, see the text.

\section{References}

1. K. Nakashima, K. Suetsugu, S. Akiyama and K. Yoshida, J. Chromatogr., 530, 154 (1990).

2. N. Nagai, Yakugaku Zasshi, 13, 901 (1893).

3. T. Kawasaki, M. Maeda and A. Tsuji, J. Chromatogr., 328, 121 (1985).

4. H. Yuki, Y. Azuma, N. Maeda and H. Kawasaki, Chem. Pharm. Bull., 36, 1905 (1988).

5. K. Hayakawa, N. Imaizumi, H. Shikura, E. Minogawa, N. Takayama, H. Kobayashi and M. Miyazaki, J. Chromatogr., 515, 459 (1990).

6. N. Takayama, H. Kobayashi and A. Tsuji, Eisei Kagaku, 30, 14 (1984).

7. T. Nagai and S. Kamiyama, J. Chromatogr., 525, 203 (1990).

8. N. C. Jain, T. C. Sneath and R. D. Budd, Clin. Chem., 20,1460 (1974).

9. M. Terada, T. Yamamoto, T. Yoshida, Y. Kuroiwa and S. Yoshimura, J. Chromatogr., 237, 285 (1982).

10. M. Terada, S. Yoshimura, T. Yamamoto, T. Yoshida and Y. Kuroiwa, Eisei Kagaku, 29, 143 (1983).

11. T. Yamamoto, M. Terada, S. Yoshimura, T. Sato, H. Kitagawa, T. Yoshida, K. Aoki and Y. Kuroiwa, Eisei Kagaku, 27, 331 (1981).

(Received March 27, 1991) (Accepted July 9, 1991) 